\section{New trends in drug treatment of heart failure in old age}

Klara Komici, ${ }^{1}$ Leonardo Bencivenga, Angela Spezzano, ${ }^{2}$ Pierangela Nocella, ${ }^{2}$ Graziamaria Corbi, ${ }^{1}$ Nicola Ferrara, ${ }^{2,3}$ Giuseppe Rengo ${ }^{2,3}$

${ }^{1}$ Department of Medicine and Health Sciences, University of Molise, Campobasso; ${ }^{2}$ Division of Geriatrics, Department of Translational Medical Sciences, Federico II University of Naples, Naples; ${ }^{3}$ Istituti Clinici Scientifici Maugeri SpA, Società Benefit, IRCCS, Istituto Scientifico di Telese, Terme (BN), Italy

\begin{abstract}
Heart failure (HF) is a complex clinical syndrome, with high prevalence in the elderly. The World Heath Organization (WHO) predicts that by 2050 the population aged over 80 years will account around 400 million, reflecting that HF will still represent a major public health concern. Improved management of cardiovascular diseases and HF, together with the increased life expectancy explains, at least in part, the high prevalence of HF especially in the elderly. Beside the canonical therapy for $\mathrm{HF}$ failure, including angiotensin converting enzyme inhibitors, angiotensin receptor blockers, beta-blockers and aldosterone antagonists, new potential and promising therapies, such as sacubitril/valsartan, iron deficiency treatment and serelaxine, are emerging also in elderly HF patients. In this review we focus on the classical recommended HF therapy and the possible application of new trends in elderly.
\end{abstract}

\section{Heart failure in elderly population}

Heart failure (HF) is a complex clinical syndrome, whose incidence and prevalence are higher among elderly compared to younger subjects; in over 80 years patients, it approaches $10 \%$. The geriatric population is also characterized by worse outcome, with a mean survival time of 2.5 years from diagnosis. ${ }^{1-3}$ The World Heath Organization (WHO) predicts that by 2050 the population aged over 80 years will account of 400 million people, suggesting that HF will still represent a major public health concern., Improved management of cardiovascular diseases and HF, together with the increased life expectancy may in part explain the high prevalence of HF especially in the elderly. However, most of the clinical trials, focusing on new potential therapies in HF, underrepresent the elderly population, leaving clinicians with very few evidence-based recommendations in this population. The reasons why these patients are generally not included in the clinical trials are mainly related to the presence of other comorbidities, impaired renal function, and different profile of drug tolerance and increased like hood of drug tolerance. These conditions, together with the aging process, influence the decision making in drug therapy prescription. ${ }^{6,7}$ However, it is also well established, that preventive therapies and medical advances are a key contributor to the extension of lifespan and to successful ageing. Aging is mainly defined by the chronological age, the subjects aged 65 years and over are usually classified as elderly. However, this is only a traditional threshold since a clear measurement of aging process is complex. Comorbidities, lifestyle, and genetics are some of the important issues to considered in the heterogeneity observed in elderly population. ${ }^{8}$ Regardless the definition of elderly, the extended lifespan may induce a longer exposure to risk factor that can lead to HF development. In addition, the age-related modification of cardiac structure and function, including impaired diastolic function and increased ventricular mass, make more prone the development of HF in elderly. The cellular pathophysiology of the ageing heart is defined by several mechanisms, such as cardiomyocyte necrosis, apoptosis and hypertrophy, enhancement of cardiac fibrosis, up-regulation of renin-angiotensine aldosterone axis and down regulation of beta-adrenergic system. ${ }^{9,10}$ These changes predispose to the development of HF with preserved systolic function; moreover, chronic inflammation and vascular stiffness facilitate the atherosclerotic process, leading to ischemic heart disease and development of HF with reduced systolic function.

\section{Heart failure treatment in elderly}

Efficacy and safety of angiotensin converting enzyme inhibitors (ACE-inhibitors) in elderly have been confirmed by important clinical trials including: Survival And Ventricular Enlargement (SAVE), The Trandorapril Cardiac Evaluation (TRACE), Studies Of Left Ventricular Dysfunction (SOLVD) and Assessment of Treatment with Lisinopril and Survival (ATLAS). ${ }^{11-14}$ Although ACE-inhibitors are strongly rec-
Correspondence: Giuseppe Rengo,

Department of Translational Medical Sciences, University of Naples Federico II, via Sergio Pansini 5, 83131 Naples, Italy. Tel.: +39.081.7462339 -

Fax: +39.081 .7462339$

E-mail: giuseppe.rengo@unina.it

Key words: Heart failure; elderly; therapy; outcome.

Contributions: GR, KK, contributed to the review conception and design; KK, LB, SA, $\mathrm{NP}$, conducted the literature searches and reviewed the identified articles; GC, NF, provided critical analysis; GC, GR, revised the manuscript for intellectual content. All authors read and approved the final manuscript.

Conflict of interest: the authors declare no potential conflict of interest.

Received for publication: 17 September 2018 Revision received: 26 October 2018.

Accepted for publication: 16 November 2018.

This work is licensed under a Creative Commons Attribution-NonCommercial 4.0 International License (CC BY-NC 4.0).

(C) Copyright K. Komici et al., 2018

Licensee PAGEPress, Italy

Geriatric Care 2018; 4:7833

doi:10.4081/gc.2018.7833

ommended, the gradual titration of dosage and the presence of side effects or drug intolerance are frequent in the elderly population with multiple comorbidities. In case of intolerance to ACE-inhibitors, angiotensin receptor blockers (ARBs) use should be considered in this population, as in the general population. Importantly, several studies did not show any negative impact of increasing age on outcomes after ARBs therapy. ${ }^{15}$

The benefits of beta-blockers in elderly HF patients have been also well documented by Study of the Effects of Nebivolol Intervention on Outcomes and Rehospitalization in Seniors with Heart Failure (SENIOR) and Metoprolol CR/XL Randomized Intervention Trial in chronic Heart Failure (MERIT-HF). ${ }^{16,17}$ In these trials, it has been demonstrated that bet-blockers use significantly reduced cardiovascular mortality and all cause mortality also in the elderly. Similar to ACE-inhibitors and ARBs, beta-blockers can cause important side effects, such as hypotension and bradycardia resulting in falls and injuries. However, a recent meta-analysis, performed in studies where elderly patients affected by hypertension were treated with ACE-inhibitors, ARBs, or beta-blockers, has shown that the 
use of these classes of drugs is not associated with a higher risk of injurious falls..$^{18}$

Although the use of aldosterone antagonists is frequently associated with the development of hyperkalaemia and renal failure, important trials as The Eplerenone in Mild Patients Hospitalization and Survival Study in Heart Failure (EMPHASIS-HF), the Randomized Aldactone Evaluation Study (RALES), and Epleronone Post-Acute Myocardial Infarction Heart Failure Efficacy and Survival Study (EPHESUS), have shown a relevant reduction in all-cause mortality, cardiovascular mortality and HF hospitalization and, importantly, these advantages are maintained in the elderly population, despite and increase in side effects. ${ }^{19,20}$

Diuretics use is recommended to reduce the signs and symptoms in patients with systolic HF, however these drugs seem to have no impact on survival. Elderly patients presenting with congestion benefit from diuretics therapy during hospitalization and they have to continue this therapy after discharge. According to Systolic Heart failure treatment with the If inhibitor ivabradine Trial (SHIFT), ivabradine use reduced either cardiovascular mortality and hospitalization rate and no differences were observed in patients older than 65 years. ${ }^{21}$

Regarding implantable cardioverter defibrillator (ICD) and cardiac resynchronization therapy (CRT) use in the elderly, several studies have reported inconsistent results. For example, the Danish Study to Assess the Efficacy of ICDs in Patients with Non-ischemic Systolic Heart Failure on Mortality (DANISH), implanted as primary prevention, has reported no benefit after ICD implantation in patients of more than 60 years old. Subgroup analysis of DANISH study showed that sudden cardiac death rate was similar in patients younger or older than 70 years, whereas non sudden cardiac death rate was significantly higher in elderly patients. ${ }^{22}$ Other studies did not report any interaction of age in the effectiveness of ICD therapy, however it should be mentioned that mostly of them recruit patients from 18 to 80 years old and patients over 80 , are excluded. Of interest, recent meta-analyses have reported no differences in overall survival between young and elderly patients after ICD implantation and, in elderly HF patients ICD therapy shows a significant higher survival compared to non implantation. ${ }^{23,24}$ Because of the low number of elderly patients included in the CRT trials, data regarding the benefit of this therapy in elderly are limited. However, some sub-analysis of studies and observational studies have reported that left ventricular ejection fraction after CRT implantation ameliorates in elderly subjects similarly to younger patients. ${ }^{25}$

\section{New trends in heart failure treatment in the elderly}

\section{Sacubitril/valsartan}

Over-activity of Renine-AngiotensinAldosterone axis is one of the most important features of chronic HF and its blockade represents a milestone in the armamentarium of HF therapy. Recently, ARB blockers combined with a neprilysin inhibitor (sacubitril) has emerged as an effective therapy in patients with HF and with an left ventricular ejection fraction of less than $35 \%$. Neprilysin is an endopeptidase responsible for the degradation of natriuretic peptides, adrenomedullin and bradykinin. Circulating natriuretic peptide, through activation of natriuretic peptide receptors, generate cGMP, resulting in myocardial relaxation, attenuation of left ventricular remodelling, enhanced diuresis and natriuresis and reduce the levels of renin and aldosterone secretion. The efficacy and safety of this drug has been described in Prospective comparison of ARNi with ACEi to Determine Impact on Global Mortality and morbidity in HF trial (the PARADIGM-HF trial), which showed that sacubitril/valsartan use was associated with a significant reduction of all cause mortality, cardiovascular mortality and HF hospitalization rate compared to enalapril. Furthermore, the neuro-hormonal profile of patients receiving sacubitril/valsartan displayed a significant reduction in circulating NT-pro-BNP levels. Despite relevant potential side effects, including hypotension and hyperkalaemia, the benefit of this therapy on survival was maintained in elderly $\mathrm{HF}$ patients and no relevant interaction between age and side effects have been reported. ${ }^{26}$

Since neprilysin is also involved in beta-amyloid clearance pathway, several concerns have been raised considering that inhibition of this enzyme could influence the degradation of beta-amyliod in the central nervous system, leading to dementia development. Regarding to this concern, data from different trials, have been analysed. ${ }^{27}$ Considering the cognitive performance no evidence of increased dementia risk has been found during a median follow-up of 2.25 years. It should be mentioned that in these studies, dementia, cognitive disorders or delirium were identified as anamnestic data, while specific tools to evaluate cognitive performance are currently used in ongoing trials. Finaly, a longer follow-up will help to definitively exclude the risk of dementia development.

\section{Iron supplements}

As we mentioned above, extra-cardiac comorbidities complicate HF progression.
Iron deficiency has been reported as a frequent comorbidity in $\mathrm{HF}$, with a prevalence ranging from $37 \%$ to $50 \%$ in European countries and up to $61.3 \%$ in USA. Mechanisms underlying iron deficiency in HF are still unclear but the reduced supply in the reticuloendothelial system together with the reduced gastro-intestinal absorption are considered to play an important role. Of note, in elderly patients, which have an high prevalence of renal function impairment, malnutrition, sarcopenia, frailty and chronic inflammatory disease, there is an higher probability to develop iron deficiency. ${ }^{28}$

The Ferric Carboxymaltose Evaluation on Performance in Patients with Iron Deficiency in Combination with Chronic Heart Failure (CONFIRM-HF) trial has demonstrated that treatment with intravenous iron supplements results in improved functional capacity and reduced symptoms in HF patients. The Ferinject Assessment in Patients with Iron Deficiency and Chronic Heart Failure (FAIR-HF trial) has reported that iron supplements in HF patients is safe and preserves renal function. Sub-analysis of these data has reported similar benefits also in elderly aged above 69 years. ${ }^{29}$

\section{Serelaxin}

Relaxin is a human hormone mainly produced by corpus luteum and placenta during pregnancy. Relaxin is known to interact with a $\mathrm{G}$ protein-coupled receptor, inducing cyclic adenosine monophosphate (cAMP) production and nitric oxide release. Additionally, relaxin upregulates the activity of vascular matrix metalloproteinase-2 influencing cardiac remodelling. Based on relaxin pharmacological properties it has been postulated that this drug increases cardiac output and arterial compliance. In Serelaxin, recombinant human relaxin-2, for treatment of acute heart failure (RelaxAHF trial), recombinant human relaxin-2 peptide hormone has been administered in HF patients to test its effect on dyspnea. This was a Phase 3 study where patients of 18 years and older have been enrolled. Data from this study have reported that use of serelaxin resulted in dyspnoea relief, increase in 180 days survival rate, but it failed to show any improvement in re-hospitalization or survival at 60 days. ${ }^{29}$ Furthermore, from the subgroup analysis, a significant reduction of mortality vs placebo has been observed in patients aged $>75$ years. However, other ongoing studies to prove efficacy of this drug are proceeding. Relax-AHF 2 study is designed to evaluate the role of intravenous infusion of serelaxin in addition to standard therapy in patients 
with acute HF. Primary endpoint are focusing on dyspnoea, cardiovascular and all cause mortality. ${ }^{30}$

\section{Left ventricular assist devices}

Left ventricular assist devices (LVAD) implantation in end-stage heart failure is mainly applied as bridge to transplantation or destination therapy. The restriction of heart transplantation in patients aged more than 65 year older, the high frequency of comorbidities among elderly patients and first diagnosis of HF in elderly subjects are responsible for the increase in the number of patients receiving LVAD as destination therapy (up to $45.7 \%$ ). ${ }^{31}$ Data from the Interagency Registry for Mechanically Assisted Circulatory Support) Profiling Identifies Ambulatory Patients at High Risk on Medical Therapy After Hospitalizations for Heart Failure (INTERMACS), report that from 2003 till 2014, the percentage of LVADs implantation in patients aged $>75$ years rose from $3 \%$ to $11 \%$. In the same period, over the years, although elderly patients undergoing LVAD implantation were older and sicker, we have witnessed a progressive reduction in mortality, dropping from $61 \%$ in 2003 to $18 \%$ in 2014 .

Although in several studies age resulted an indipended predictor of mortality after LVAD implantation, INTERMACS registry have reported a $63 \%$ survival rate in patients aged more than 70 years. ${ }^{32}$ Even though, the survival in younger patients is significantly higher, considering this age group and compering it to elderly patients receiving only drug therapy, the results look promising. Interestingly, in a population of frail LVAD patients, according to Fried criteria, it has been observed that in $50 \%$ of them frailty status significantly decreased after 6 months of LVAD transplantation. ${ }^{33}$ Indeed, it has been described a robust improvement in physical performance of this patients after LVAD implantation. However, as reported from a recent metaanalysis, frailty significantly affects survival in LVAD recipients. ${ }^{34}$

\section{Conclusions}

Despite HF is a disease that mainly affects the elderly population, the choice of the best therapeutic strategy is always challenging in this population. However, recent clinical trials and guidelines suggest that the recommendations for HF treatment in the general population, including new promising therapies and strategies, may improve survival also in the elderly population.

\section{References}

1. Roger VL, Go AS, Lloyd-Jones et al. Heart disease and stroke statistics - 2012 update: a report from the American Heart Association. Circulation 2012;125: e2-e220.

2. McMurray JJ, Adamopoulos S, Anker $\mathrm{SD}$, et al. ESC guidelines for the diagnosis treatment of acute chronic heart failure 2012: the Task Force for the Diagnosis Treatment of Acute Chronic Heart Failure 2012 of the European Society of Cardiology. Developed in collaboration with the Heart Failure Association (HFA) of the ESC. Eur J Heart Fail 2012;14:803-69.

3. Lindenfeld J, Albert NM, Boehmer JP, et al. HFSA 2010 comprehensive heart failure practice guideline. J Card Fail 2010;16:e1-194.

4. World Health Organisation. 10 facts on ageing and the life course; 2012. Available from: http:/www.who.int/features/factfiles/ageing/ageing_facts/en/in dex.html Accessed: 02/02/2014.

5. Lazzarini V, Mentz RJ, Fiuzat M, et al. Heart failure in elderly patients: distinctive features and unresolved issues. Eur J Heart Fail 2013;15:717-23.

6. Corbi G, Ambrosino I, Komici K, et al. Gender differences in response to therapy for cardiovascular diseases. Curr Pharmacogenom Person Med 2015;1: 14-26.

7. Bencivenga L, Grieco FV, Femminella GD, et al. Management and treatment of cardiovascular diseases in the elderly. Curr Pharmacogenom Person Med 2015;1:5-13.

8. Singh S, Bajorek B. Defining 'elderly' in clinical practice guidelines for pharmacotherapy. Pharm Pract (Granada) 2014; 12:489.

9. de Lucia C, Eguchi A, Koch WJ. New insights in cardiac $\beta$-adrenergic signaling during heart failure and aging. Front Pharmacol 2018;9:904.

10. Ferrara N, Komici K, Corbi G, et al. $\beta$ adrenergic receptor responsiveness in aging heart and clinical implications. Front Physiol 2014;4:396.

11. Køber L, Torp-Pedersen C, Carlsen JE, et al. A clinical trial of the angiotensinconverting-enzyme inhibitor trandolapril in patients with left ventricular dysfunction after myocardial infarction. N Engl J Med 1995;333:1670-6.

12. Packer M, Poole-Wilson PA, Armstrong $\mathrm{PW}$, et al. Comparative effects of lowand high doses of the angiotensin-converting enzyme inhibitor, lisinopril, on morbidity and mortality in chronic heart failure. Circulation 1999;100:2312-18.

13. The SOLVD Investigators. Effect of enalapril on survival in patients with reduced left ventricular ejection fractions and congestive heart failure. $\mathrm{N}$ Engl J Med 1991;325:293-302.

14. Pfeffer MA, Braunwald E, Moy'e LA, et al. Effect of captopril on mortality and morbidity in patients with left ventricular dysfunction after myocardial infarction. Results of the Survival and Ventricular Enlargement Trial. N Engl J Med 1992;327:669-77.

15. Guerra F, Brambatti M, Matassini MV, et al. Current therapeutic options for heart failure in elderly patients. Biomed Res Int 2017;2017:1483873.

16. Ang HT, Lim KK, Kwan YH, et al. A systematic review and meta-analyses of the association between anti-hypertensive classes and the risk of falls among older adults. Drugs Aging 2018 [Epub ahead of print].

17. Metoprolol CR/XL Randomised Intervention Trial in-Congestive Heart Failure (MERIT-HF). Lancet 1999;353: 2001-7.

18. Flather MD, Shibata MC, Coats AJ,S et al. Randomized trial to determine the effect of nebivolol on mortality and cardiovascular hospital admission in elderly patients with heart failure (SENIORS). Eur Heart J 2005;26:215-25.

19. Pitt B, Zannad F, Remme WJ, et al. The effect of spironolactone on morbidity and mortality in patients with severe heart failure. N Engl J Med 1999;341: 709-17.

20. Zannad F, McMurray JJ, Krum H, et al. Eplerenone in patients with systolic heart failure and mild symptoms. N Engl J Med 2011;364:11-21.

21. Das D, Savarese G, Dahlström U, et al. Ivabradine in heart failure: the representativeness of SHIFT (Systolic Heart Failure Treatment With the IF Inhibitor Ivabradine Trial) in a broad population of patients with chronic heart failure. Circ Heart Fail 2017 [Epub ahead of print].

22. Elming MB, Nielsen JC, Haarbo J, et al. Age and outcomes of primary prevention implantable cardioverter-defibrillators in patients with nonischemic systolic heart failure. Circulation. 2017;136:1772-80

23. Kong M, Al-Khatib M, Sanders GD, et al. Use of implantable cardioverterdefibrillators for primary prevention in older patients: A systematic literature review and meta-analysis. Cardiol J 2011;18:503-14.

24. Brambatti M, Guerra F, Matassini MV, et al. Cardiac resynchronization therapy improves ejection fraction and cardiac 
remodelling regardless of patients' age. Europace 2013;15:704-10.

25. Pitt B, Remme W, Zannad F, et al. Eplerenone, a selective aldosterone blocker, in patients with left ventricular dysfunction after myocardial infarction. N Engl J Med 2003;348:1309-21.

26. McMurray JJW, Packer M, Desai AS, et al. Angiotensinneprilysin inhibition versus enalapril in heart failure. N Engl J Med 2014;371:993-1004.

27. Cannon JA, Shen L, Jhund PS, et al. PARADIGM-HF Investigators and Committees. Dementia-related adverse events in PARADIGM-HF and other trials in heart failure with reduced ejection fraction. Eur J Heart Fail 2017;19: 129-37.
28. Von Haehling S, Anker MS, Jankowska EA, et al. Anemia in chronic heart failure: Can we treat? What to treat?. Heart Fail Rev 2012;17:203-10.

29. Ponikowski P, van Veldhuisen DJ, Comin-Colet J, et al. CONFIRM-HF Investigators. Beneficial effects of long-term intravenous iron therapy with ferric carboxymaltose in patients with symptomatic heart failure and iron deficiency. Eur Heart J 2015;36:657-68.

30. Teerlink JR, Cotter G, Davison BA, et al. RELAXin in Acute Heart Failure (RELAX-AHF) Investigators. Serelaxin, recombinant human relaxin-2, for treatment of acute heart failure (RELAXAHF): a randomised, placebo-controlled trial. Lancet 20135;381:29-39.
31. Teerlink JR, Voors AA, Ponikowski P, et al. Serelaxin in addition to standard therapy in acute heart failure: rationale and design of the RELAX-AHF-2 study. Eur J Heart Fail. 2017;19:800-9.

32. Kirklin JK, Naftel DC, Pagani FD, et al. Seventh INTERMACS annual report: 15,000 patients and counting. J Heart Lung Transplant 2015;34:1495-504.

33. Kirklin JK, Naftel DC, Pagani FD, et al. SixthINTERMACS annual report: a 10,000-patient database. J Heart Lung Transplant 2014;33:555-64.

34. Maurer MS, Horn E, Reyentovich A, et al. Can a left ventricular assist device in individuals with advanced systolic heart failure improve or reverse frailty? J Am Geriatr Soc 2017;65:2383-90. 